\title{
ARTICLE
}

\section{Experiences on radioactivity handling for mercury target system in MLF/J-PARC}

\author{
Tetsuya Kai*, Yoshimi Kasugai, Motoki Ooi, Hiroyuki Kogawa, Katsuhiro Haga, Hidetaka Kinoshita, Masakazu Seki \\ and Masahide Harada \\ Japan Atomic Energy Agency, 2-4 Shirakata, Tokai-mura, Naka-gun, Ibaraki-ken, 319-1195, Japan
}

\begin{abstract}
Various kinds of radioactivity are produced via spallation reactions by injecting high energy intense proton beam $(3 \mathrm{GeV}, 1 \mathrm{MW})$ on a mercury target in the Materials and Life science experimental Facility of J-PARC. Radioactivity handling strategy was designed based on estimations by using particle transport calculation codes NMTC/JAM, MCNP/4C and an induced radioactivity calculation code DCHAIN-SP 2001. The radiation around a mercury circulation system was estimated to be reduced by draining mercury into a shielded tank. The volatile radioactivity produced in mercury was designed to be processed by an off-gas process system. Practical experiences through facility operations were quite different from these strategies. The radiation around the mercury circulation system increased after draining mercury. Mercury radioactivity has not been detected so far in a mercury absorber of the off-gas process system while xenon-127 trapped in the absorber increased radiation dose around the absorber. The amount of tritium release was much higher at a target chamber replacement than that expected from the preceding flushing process. This paper describes how the unexpected issues have been tackled and what was understood for the differences.
\end{abstract}

Keywords: mercury target; radioactivity; xenon-127; tritium; measurement; spallation products; off-gas process system; PHITS; DCHAIN-SP 2001; intense neutron source

\section{Introduction}

The Japanese spallation neutron source in the Materials and Life science experimental Facility (MLF) of the Japan Proton Accelerator Complex (J-PARC) [1] started operation in 2008. The intense proton beam of 3 $\mathrm{GeV}$ and $1 \mathrm{MW}$ is expected to irradiate a mercury target in a target-moderator-reflector assembly (TMRA) to provide neutrons mainly for various neutron-scattering experiments. Large amount of radioactivity is also produced in the neutron source with the proton beam irradiation. Handling strategy of the radioactivity was developed based on calculations [2] by using a code system consisting of NMTC/JAM [3] (being upgraded to PHITS [4] later), MCNP/4C [5] and DCHAIN-SP 2001 [6] codes. It was assumed that mercury in the target chamber (0.8-litter) was irradiated continuously, and that the calculated radioactivity was diluted by total volume of mercury $\left(2.1 \mathrm{~m}^{3}\right)$. For example the radiation dose rate of $1-\mathrm{ml}$ mercury was estimated to give $0.13 \mathrm{mSv} / \mathrm{h}$ at $1 \mathrm{~m}$ from the source in case of the 10-day cooling after 30 years $(5000 \mathrm{~h} / \mathrm{y})$ irradiation at $1 \mathrm{MW}$. The contributions of mercury-203 $\left({ }^{203} \mathrm{Hg}\right)$, iridium-188 $\quad\left({ }^{188} \mathrm{Ir}\right)$ and osmium-185 ( $\left.{ }^{185} \mathrm{Os}\right)$ were $48 \%, \quad 17 \%$ and $9 \%$, respectively. Hands-on access around irradiated mercury was discussed based on this estimation. The radiation dose from the cooling water of TMRA was also

*Corresponding author. Email: tetsuya.kai@j-parc.jp calculated. It was estimated to decrease to one-thousandth by 2-minute cooling. Decay tanks were introduced to reduce radiation damages of pump components and costs of shielding by taking the decay time. The calculations of radioactivity played important roles in the facility design works.

The proton beam power was $4 \mathrm{~kW}$ at the beginning of the facility operation, and then increased to 20,100 and $200 \mathrm{~kW}$ in a step-by-step manner. Experiences of radioactivity handing have been accumulated with the increase of the proton beam power. Most of large discrepancies from design concepts were found in behavior of radioactivity produced in mercury. This was natural since there had been few experiences of liquid metal handling as spallation target material. In this paper, the authors describe experiences of radioactivity handling in MLF, those will be useful for design works for future facilities with a liquid metal spallation target.

\section{Design concept for mercury radioactivity handling}

\subsection{Radiation from mercury circulation system}

A mercury-circulation system was equipped to remove heat generated by the proton irradiation. Most components were designed to be maintained using remote handling tools in a hot cell considering high radiation dose of mercury. Mercury in the circulation 
system was designed to be drained to tanks surrounded by $30-\mathrm{cm}$ thick iron-shields to reduce radiation for hands-on maintenance of many ordinary equipments such as cameras, bulbs, air filters, cranes, etc. The radiation dose rate from the mercury circulation system was confirmed to be acceptable by estimation with a final design after extensive considerations to minimize remaining mercury after draining and local shields to components having large amount of remaining mercury. This design concept was expected to bring about large advantages in time, cost, and flexibility concerning to maintenance in the hot cell [7].

\subsection{Off-gas process system}

Figure 1 shows a conceptual diagram of the mercury circulation system and the off-gas process system. The mercury circulation system has a surge tank to pressurize mercury by helium cover gas. Volatile elements in the spallation products were expected to be accumulated in the cover gas. Note that iodine was assumed to remain in mercury by composing mercury iodine. Although mercury was designed to be used as long as the facility lifetime, a mercury target-chamber needed to be replaced twice a year due to radiation damages by the proton beam irradiation. The radioactivity in the cover gas had to be processed properly before replacing the target chamber. An off-gas process system was introduced to reduce mercury vapor for contamination prevention, tritium and xenon-127 $\left({ }^{127} \mathrm{Xe}\right)$ to obey the regulation by law. The cover gas is transferred to a gas holder through a mercury absorber, and is kept in the holder about a year mainly for decaying ${ }^{127} \mathrm{Xe}\left(\mathrm{T}_{1 / 2}=36.4\right.$ day). Tritiated water (HTO) is trapped by a molecular sieve in a tritium removal system before exhausting the gas to the environment. If necessary, tritiated hydrogen (HT) in the cover gas is converted to HTO by an oxidation catalyst in the tritium removal system [8].

\section{Experiences in radioactivity handling}

\subsection{Radiation dose after mercury drain}

Radiation dose rate around the mercury circulation

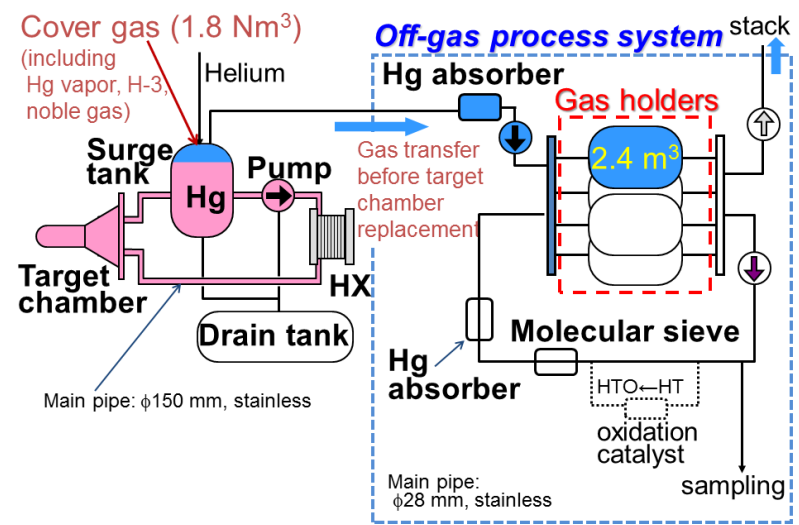

Figure 1. A conceptual diagram of mercury circulation system and off-gas process system. system was measured at 40 days after the first beam operation of $130 \mathrm{kWh}$. It was assumed that spallation products distribute homogeneously in mercury to calculate the radiation dose rate. The highest dose rate of $1 \mathrm{mSv} / \mathrm{h}$ was measured at a surface of mercury loaded straight pipe. The dose rates at surfaces of pipes increased after draining mercury by a factor of 2 in average. This was an opposite behavior to the expectation. The measured dose rates were much higher than the calculated values.

Gamma-ray spectroscopy was carried out during mercury drain to understand the unexpected phenomena by using a portable high purity germanium detector (HPGe), resulting that the gamma-ray from ${ }^{203} \mathrm{Hg}$ decreased just after mercury draining, while those from ${ }^{185}$ Os and ${ }^{188} \mathrm{Ir}$ (daughter of platinum-188) increased. It was indicated that some spallation products adhering to the inner surface of the circulation system components, and mercury acted as a shield against them [9]. The fact that shielding effects were higher in the lower gamma-ray energy is consistent to the above conclusion.

To obtain straightforward explanation the inner surface of pipe giving the highest radiation was smeared by small pieces of paper, and remaining mercury was taken from the circulation system. Gamma-ray from these specimens was measured by an HPGe detector. Twelve nuclei $\left({ }^{203} \mathrm{Hg},{ }^{189} \mathrm{Ir},{ }^{188} \mathrm{Ir},{ }^{188} \mathrm{Pt},{ }^{185} \mathrm{Os},{ }^{175} \mathrm{Hf},{ }^{173} \mathrm{Lu}\right.$, ${ }^{172} \mathrm{Lu},{ }^{146} \mathrm{Eu},{ }^{113} \mathrm{Sn},{ }^{110 \mathrm{~m}} \mathrm{Ag},{ }^{88} \mathrm{Y}$ ) were identified in the mercury specimen. The derived values of radioactivity agreed with the calculation in the one order of magnitude although large uncertainty was unavoidable due to ambiguity of detection efficiency by self absorption and distribution of spallation products in the mercury specimen. Same nuclei except ${ }^{203} \mathrm{Hg}$ and ${ }^{110 \mathrm{~m}} \mathrm{Ag}$ were also identified in the smeared specimen with almost same ratio to calculated yields. The ratio of the measured radioactivity to the calculated one was $24 \mathrm{ppm}$ in average [10]. To guess the maximum radioactivity adhering inner surface followings were assumed: the smeared area of 10 by $10 \mathrm{~cm}^{2}$, the decontamination coefficient of $10 \%$ for smearing and the total inner surface area of $35 \mathrm{~m}^{2}$. The derived ratio of adhering radioactivity to calculated one was 0.8 in average. It was indicated that the radiation dose from these adhering ten nuclei could be roughly estimated by assuming most

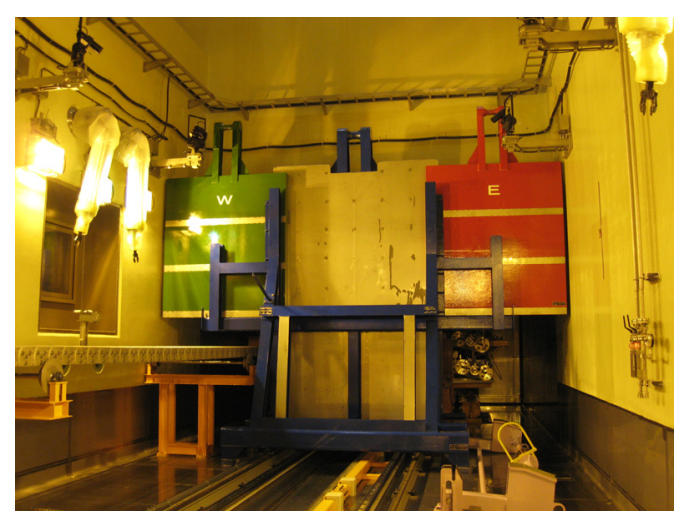

Figure 2. Movable local shield to cover mercury circulation system to reduce radiation in hot cell. 
$(80 \%)$ of calculated radioactivity distributed homogeneously on inner surface of components.

Most of radiation from the mercury circulation system was emitted from pipes since local shields had been equipped to all components having mass of remaining mercury. The mercury circulation system is too complicated to add local shield later. Thus, huge moveable shields to cover the mercury circulation system were developed as shown in Figure 2.

\subsection{Mercury absorber and xenon}

The mercury absorbers (sulfur added charcoal of 10 litters) were installed to reduce mercury vapor. Gas in the mercury circulation system transferred through one of absorbers except such emergency case as the failure of mercury containment. As a result of gamma-ray spectroscopy mercury isotopes have never been detected in the mercury absorber so far. However it is not concluded that mercury vapor dose not reach to the off-gas system through a long pipe because it was difficult to measure the gamma-ray from ${ }^{203} \mathrm{Hg}$, the dominant isotope in mercury in viewpoint of radioactivity, due to high background from ${ }^{127} \mathrm{Xe}$ trapped in the mercury absorber. The gamma-ray energy of $279 \mathrm{keV}$ from ${ }^{203} \mathrm{Hg}$ was lower than that of $375 \mathrm{keV}$ from ${ }^{127} \mathrm{Xe}$, and the half life of ${ }^{203} \mathrm{Hg}$ (47 days) is not so different from that of ${ }^{127} \mathrm{Xe}$ (36 days).

High radiation around the off-gas process system was brought about from ${ }^{127} \mathrm{Xe}$ trapped in the mercury absorber. Although the highest radiation position on the mercury absorber moved gradually from upstream to downstream during gas transfer, it was inferred to take long time to decrease the radiation dose by spontaneous release of ${ }^{127} \mathrm{Xe}$. A heater was put to surround the mercury absorber to promote release of ${ }^{127} \mathrm{Xe}$. It is operated at 150 degrees Celsius. A local shield $(10 \mathrm{~cm}$ iron) was put around the mercury absorber since some amount of ${ }^{127} \mathrm{Xe}$ was trapped temporary even though the absorber was heated. The place to put the shield had been planned originally for the case of accumulated mercury radioactivity gave high radiation. Figure $\mathbf{3}$ shows the variation of radiation dose rate at the mercury absorber (inside the shield) measured by a silicon

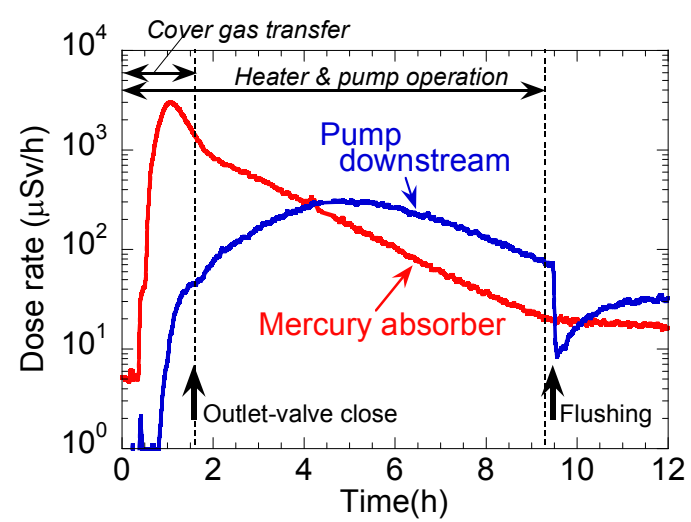

Figure 3. Time evolution of dose rate at mercury absorber and at downstream of pump after beginning of cover gas transfer. semiconductor detector during the gas transfer at 3 days after the 4-month operation with a proton beam intensity of $210-280 \mathrm{~kW}$. The gas was transferred by a pump to the off-gas process system for 1.6 hours, and then an outlet valve of the mercury circulation system was closed. The pump and the heater were operated for 9.3 hours after the beginning of gas transfer. The radiation dose rate at the mercury absorber increased up to 3 $\mathrm{mSv} / \mathrm{h}$, and decreased monotonically after closing the outlet valve. The dose rate at the downstream of the pump increased slower than that at the mercury absorber as shown in Figure $3 .{ }^{127} \mathrm{Xe}$ released from the mercury absorber remained in the pipe because there was almost no flow of gas since the pressure of the absorber and pipes was very low. The rapid depression of the radiation dose at 9.5 hours was caused by a flushing operation by helium gas. Although the radiation dose increased again after the flushing operation, it could be reduced by repeating flushing processes several times.

\subsection{Target chamber replacement}

The first replacement of the target chamber was carried out on November 2011. Based on the designed scenario the cover gas was transferred to the off-gas process system, and then mercury was drained. A certain amount of radioactivity was expected to be released when the target chamber was disconnected from the mercury circulation system. To minimize the radioactivity release in the hot cell, a purge process (inlet helium gas to the mercury circulation system and exhaust the gas to the off-gas process system) was repeated 8 times. Before disconnecting the target chamber, a proton beam incident part of the target chamber was cut to take a circular specimen for post irradiation examinations. Through the cutting hole, air flowed into the mercury circulation system which had been evacuated before the cutting. The inflow air was transferred to the off-gas system seven times before the target replacement. After connecting a new target chamber, air remaining in the circulation system was transferred, and then the helium purge process was taken place.

Figure 4 shows radioactivity evolutions of ${ }^{127} \mathrm{Xe}$ and

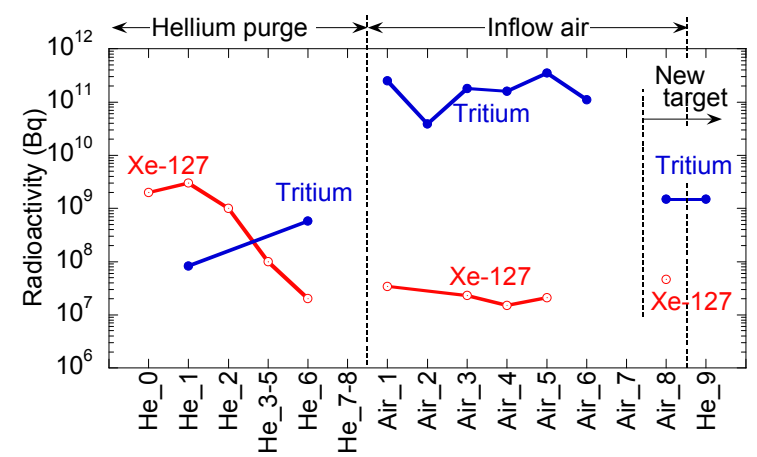

Figure 4. Time evolution of radioactivity of ${ }^{127} \mathrm{Xe}$ and tritium in transferred gas to off-gas process system. Vertical axis indicates purge IDs. For example 'He_3-5' means the plotted radioactivity was contained in the $3 \mathrm{rd}$, 4th and 5 th purge helium gas. 
tritium in each transferred gas. Radioactivity of ${ }^{127} \mathrm{Xe}$ was reduced with the purge process down to two orders of magnitudes and almost unchanged at $10^{7} \mathrm{~Bq}$ after that. This result suggested that further reduction of ${ }^{127} \mathrm{Xe}$ was difficult with the purge process. The radioactivity of tritium was below $10^{9} \mathrm{~Bq}$ in the helium purge processes. More than $10^{11} \mathrm{~Bq}$ of tritium was observed in each inflow air after the target chamber cutting. It decreased down to the order of $10^{9} \mathrm{~Bq}$ after connecting the new target chamber. It was inferred that tritium was absorbed in the inner surface of target chamber and was released via an isotope exchange reaction with water in the inflow air. It was calculated that $10^{13} \mathrm{~Bq}$ of tritium had been produced in mercury. The total measured tritium (including being released to the hot cell) was only several percent of the calculation even though a large amount of tritium was detached by the isotope exchange reaction.

The target chamber replacement was carried out after about 7-month cooling time, which was brought about by a long shutdown after the Great East Japan Earthquake. Note that ${ }^{127} \mathrm{Xe}$ radioactivity had reduced by a factor of about $2^{6}$ in the cooling time. The target chamber needs to be replaced as quickly as possible in the case of failure of a target chamber during normal operations for users. A target replacement after short cooling time would be difficult or impossible due to higher radioactivity of ${ }^{127} \mathrm{Xe}$. Moreover, a leak test is required by keeping high pressure helium gas in the mercury circulation system with a new target chamber. This large amount of gas may be impossible to be released to the environment if the cooling time is short. The number of gas holders in the off-gas process system is not enough to keep the large amount of radioactive gas. For periodic (twice a year) replacements of target chamber in near future a volume reduction system by separating xenon from helium is under development based on this experience.

Tritium was not reduced by the purge process even with wet air, and the reduction of ${ }^{127} \mathrm{Xe}$ saturated at about $1 / 100$. It was found that the purge process was effective to some extent but had limitations to reduce radioactivity in the mercury circulation system. It is unavoidable that a certain amount of radioactivity is released in the hot cell during target chamber replacement. Such additional procedures as shortening time for replacement and controlling air flow are required to minimize the release of radioactivity.

\section{Summary}

The radioactivity handling strategy had been developed based on the radioactivity calculations. Many discrepancies have been found between the design and the practical experiences especially radioactivity produced in mercury. Each problem has been tackled individually. All components including pipes of the mercury circulation system should have local shields, assuming all spallation products adhered to the inner surface of the components. The scenario of target chamber replacement should have been developed taking into account that a certain amount of tritium and ${ }^{127} \mathrm{Xe}$ was released in the air. The authors wish these experiences are effectively utilized in design of future liquid metal spallation target systems.

\section{Acknowledgements}

The authors thank staff of the radiation safety section in J-PARC for extensive assistance.

\section{References}

[1] http://www.j-parc.jp/index-e.html

[2] T. Kai, M. Harada, F. Maekawa, M. Teshigawara, C. Konno and Y. Ikeda, Induced-radioactivity in J-PARC spallation neutron source, J. Nucl. Sci. Technol. Sup. 4 (2004), pp. 172-175.

[3] K. Niita, H. Takada, S. Meigo and Y. Ikeda, High-energy particle transport code NMTC/JAM, Nucl. Instrum. Meth. B184 (2001), pp. 406-420.

[4] K. Niita, N. Matsuda, Y. Iwamoto, H. Iwase, T. Sato, H. Nakashima, Y. Sakamoto and L. Sihver, PHITS: Particle and Heavy Ion Transport Code System, Version 2.23, JAEA-Data/Code 2010-022, Japan Atomic Energy Agency, (2010).

[5] J. F. Briesmeister (Ed.), MCNP $P^{T M}-A$ General Monte Carlo N-Particle Transport Code Version 4C, LA-13709-M, Los Alamos National Laboratory, (2000).

[6] T. Kai, F. Maekawa, K. Kosako, Y. Kasugai, H. Takada and Y. Ikeda, DCHAIN-SP 2001: High Energy Particle Induced Radioactivity Calculation Code, JAERI-Data/Code 2001-016, Japan Atomic Energy Research Institute, (2001). [in Japanese]

[7] T. Kai, Y. Kasugai, M. Harada, K. Haga, H. Kogawa and F. Maekawa, Radioactivity estimation at J-PARC spallation neutron source by using the DCHAIN-SP 2001 and PHITS codes, Proc. ARIA 2008, Oct. 13-17, 2008, Paul Scherrer Institut, Switzerland, (2008), pp. 182-187, ISSN 1019-0643.

[8] S. Sakamoto (Ed), Technical Design Report of Spallation Neutron Source Facility in J-PARC, JAEA-Technology 2011-035, Japan Atomic Energy Agency, (2012), pp. 465-469.

[9] Y. Kasugai, M. Ooi and T. Kai, Gamma dose measurements and spectroscopy analysis for spallation products in JSNS mercury circulation system, Progress in Nucl. Sci. Technol. 1 (2011), pp. 501-504.

[10]T. Kai, M. Ooi, Y. Kasugai, T. Wakui, H. Kogawa, K. Haga and K. Hanano, Discussions on spallation products behaviour by measuring mercury and substances adhering inside the mercury circulation system in MLF/J-PARC, Proc. ICANS-XIX, Mar. 8-12, 2010, Grindelwald, Switzerland, (2010), ISSN 1019-6447. 